Supporting information

\title{
How Stabilizers and Reducing Agents Affect the Formation of Nanogold Amalgams
}

\author{
Nan Wang, Pengfei Cao, Houyi Ma*, Meng Lin* \\ School of Chemistry and Chemical Engineering, Shandong University, Shanda Nanlu \\ 27, Jinan, 250100, P. R. China. \\ *Corresponding authors: mlin@sdu.edu.cn (M. Lin); hyma@sdu.edu.cn (H. Ma).
}

Table of Contents

Fig. S1. The relationship between CTAB stabilized AuNRs and reducing

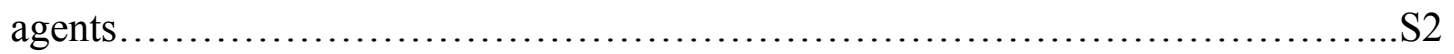

Fig. S2. LSPR shift of CTAB-stabilized AuNRs under insufficient reducing

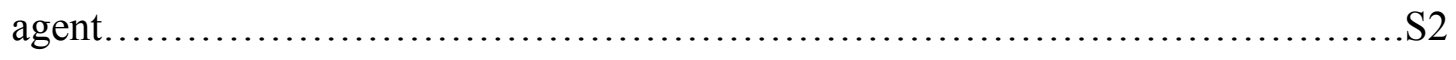

Fig. S3. HRTEM images of AuNRs at different stages.......................... 3

Fig. S4. High-resolution XPS images of $\mathrm{Au}$ and $\mathrm{Au}-\mathrm{Hg}$ amalgam nanoalloy..........S3

Fig. S5. The distribution graph of the width and length of CTAB modified gold

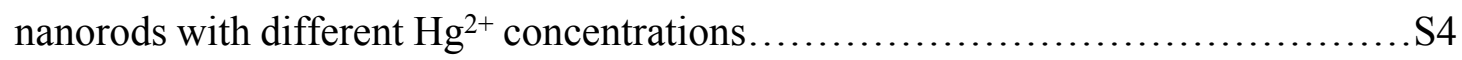

Fig. S6. Spectroscopic and XPS characterization of AuNRs modified by three different

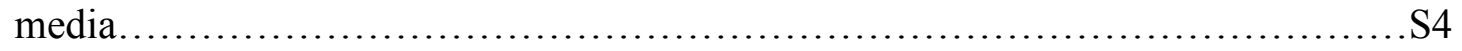

Fig. S7. UV-vis absorption spectra and TEM images of thiol-modified AuNRs under

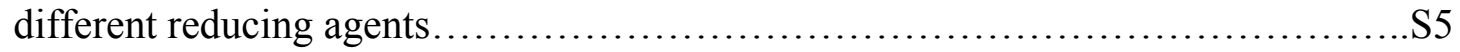

Fig. S8. SERS spectra of thiol-modified AuNRs before and after adding $\mathrm{Hg}^{2+} \ldots \ldots$.. S5 Fig. S9. UV-visible absorption spectra of gold nanooctahedra and gold nanocubewith different volumes of $\mathrm{Hg}^{2+}$ reduced by excess $\mathrm{NaBH}_{4} \ldots \ldots \ldots \ldots \ldots \ldots \ldots \ldots \ldots . . \ldots \ldots$ Table S1. Measured average zeta-potential during the reaction process of gold nanorods

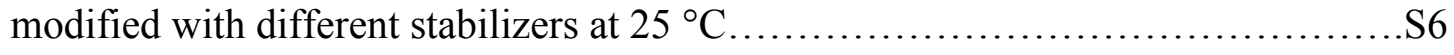



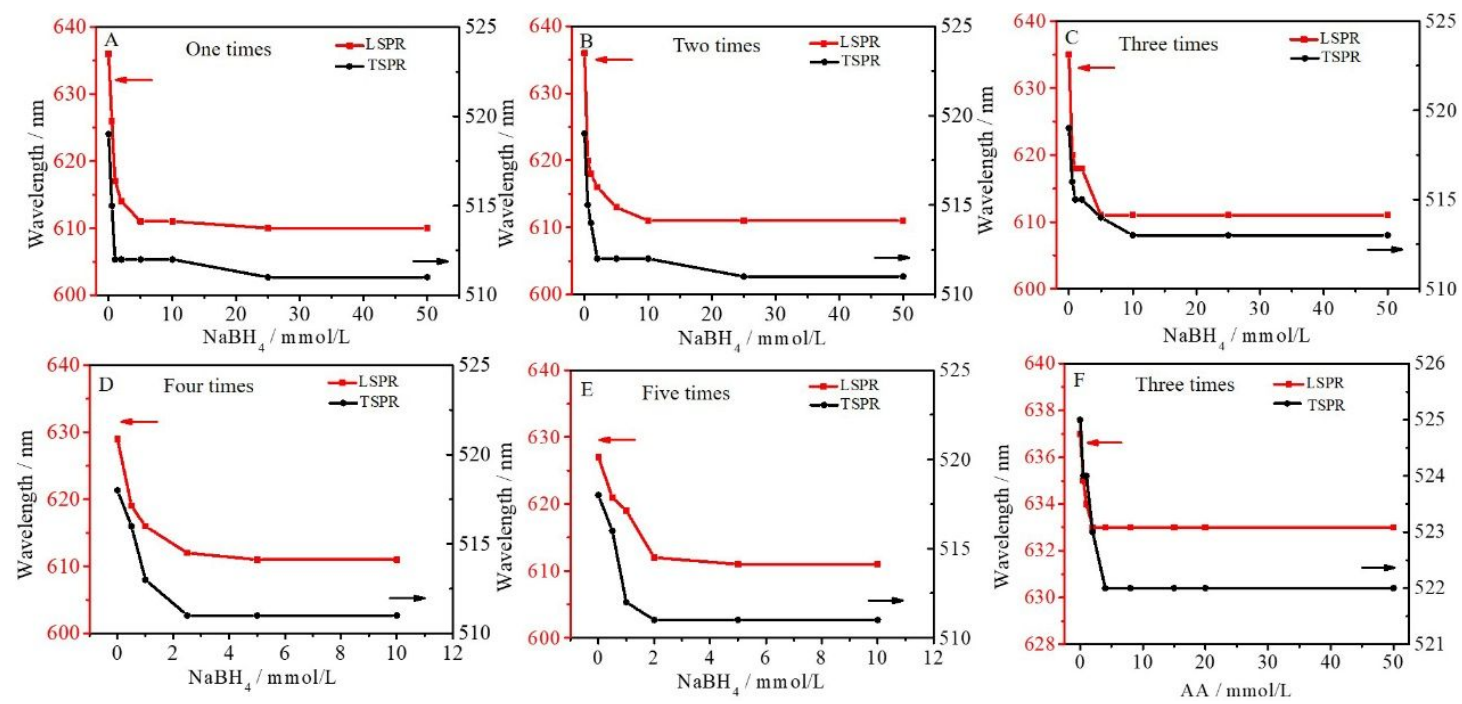

Fig. S1. Relationship between $\mathrm{CTAB}$ and $\mathrm{NaBH}_{4}$ concentration (determined by centrifugation). (A-E) The plots show the wavelength shift of the LSPR (black line) and TSPR (red line) of the AuNRs stabilized with different amounts of CTAB molecules as a function of $\mathrm{NaBH}_{4}$ concentration. (F) The plot displays the wavelength shift of the AuNRs with centrifuged three times as a function of AA content.
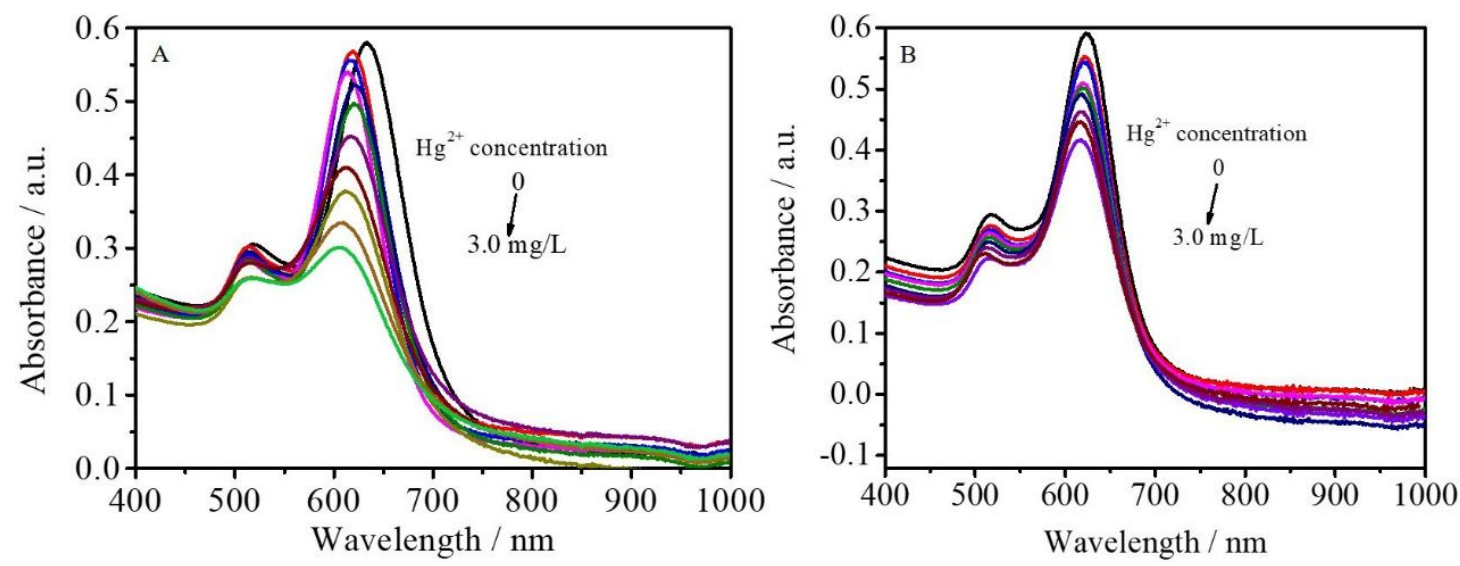

Fig. S2. (A) UV-visible spectra of the reaction between CTAB-stabilized AuNRs and different volumes of $\mathrm{Hg}^{2+}$ with the reducing agent $\mathrm{NaBH}_{4}$ content is insufficient (2.0 $\mathrm{mmol} / \mathrm{L}$ ) and (B) UV-visible spectral changes of AuNRs in the presence of AA (insufficient amount $2.0 \mathrm{mmol} / \mathrm{L}$ ) in aqueous solution. 


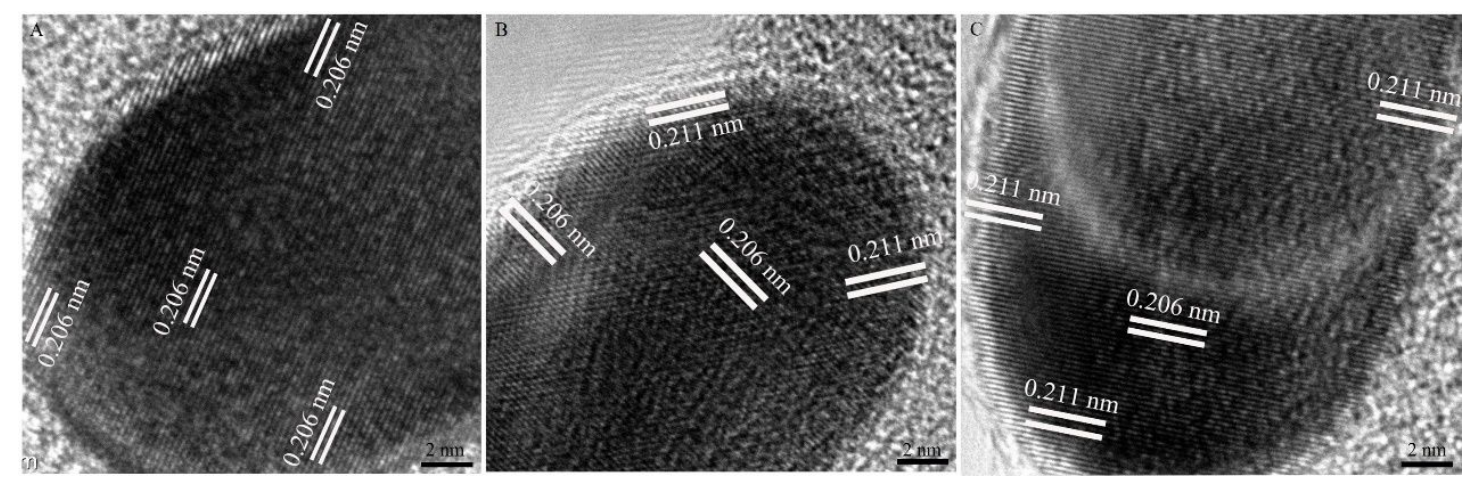

Fig. S3. HRTEM images of the (A) original CTAB-stabilized AuNRs, and the AuNRs (B) in the presence of $\mathrm{Hg}^{2+}(0.5 \mathrm{mg} / \mathrm{L})$ and $(\mathrm{C}) \mathrm{Hg}^{2+}(1.5 \mathrm{mg} / \mathrm{L})$.
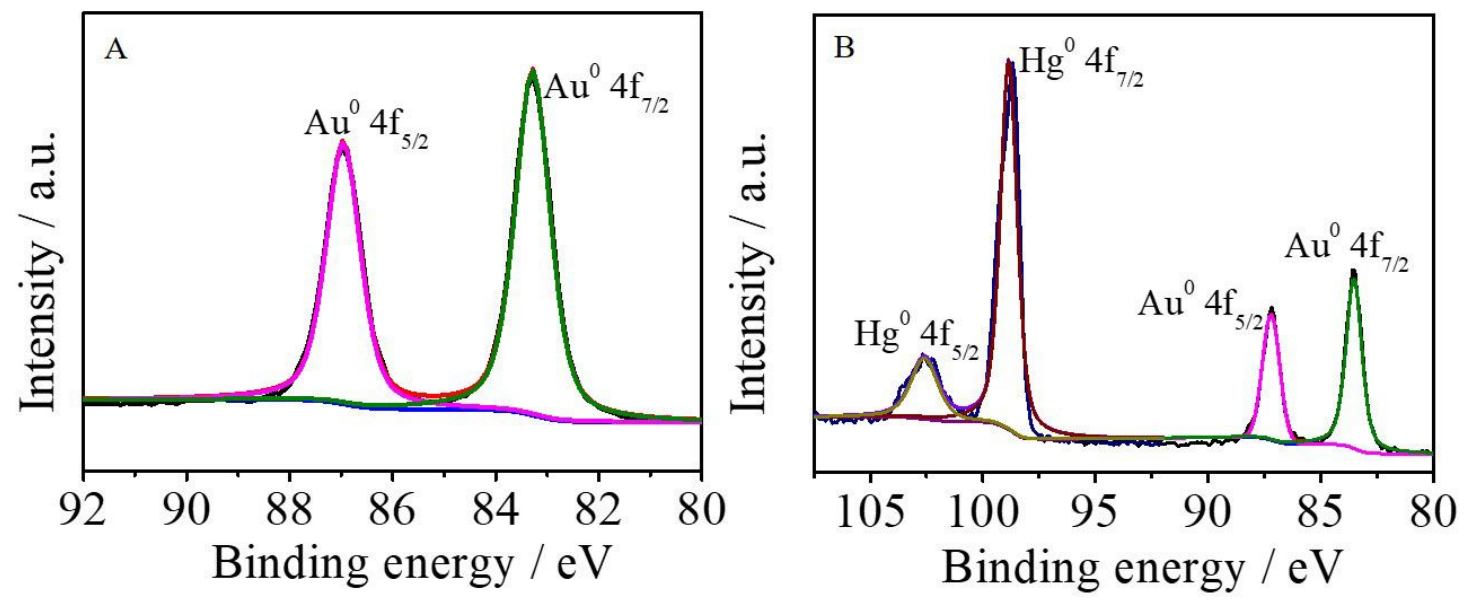

Fig. S4. High-resolution XPS images of $\mathrm{Au} 4 \mathrm{f}(\mathrm{A})$ and $\mathrm{Au}-\mathrm{Hg}$ amalgam nanoalloy (B). 

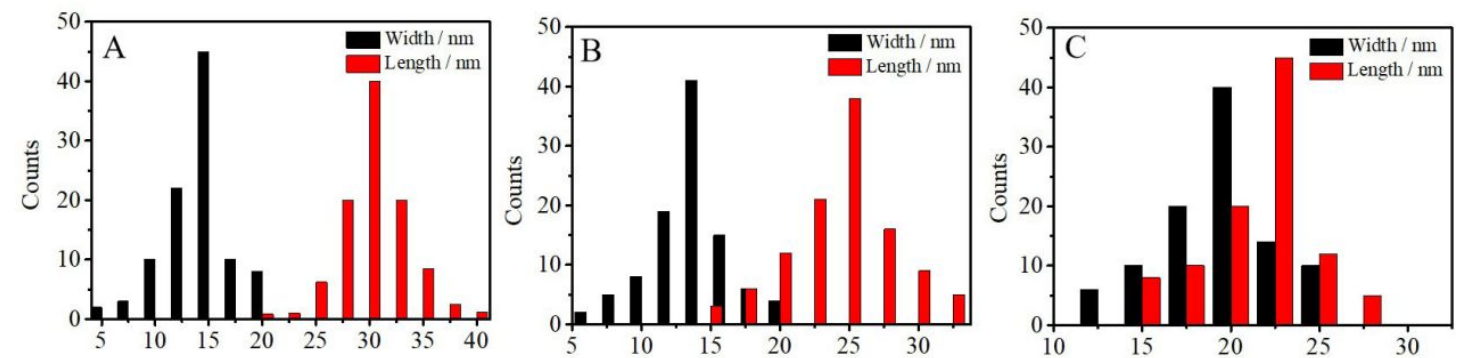

Fig. S5. The distribution graph of the width and length of CTAB modified gold nanorods with different $\mathrm{Hg}^{2+}$ concentrations (A: Initial state, $0 \mathrm{mg} / \mathrm{L}$; B: Intermediate state, $1.5 \mathrm{mg} / \mathrm{L}$; C: Final state, $3.0 \mathrm{mg} / \mathrm{L})$.

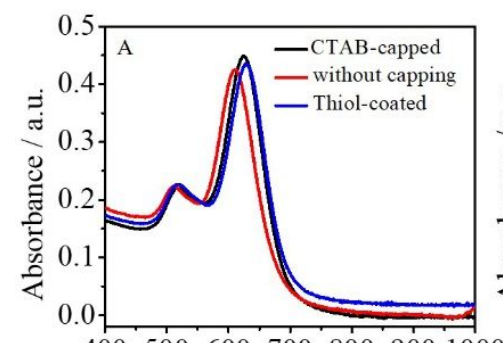

4005006007008009
Wavelength $/ \mathrm{nm}$

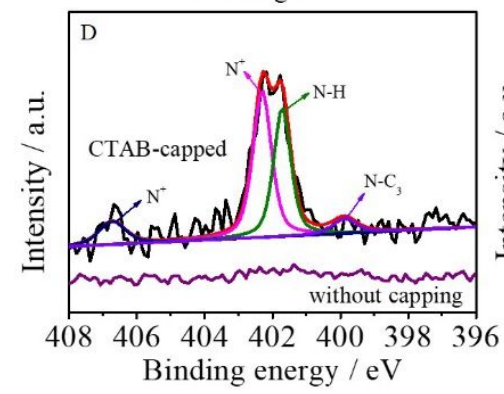

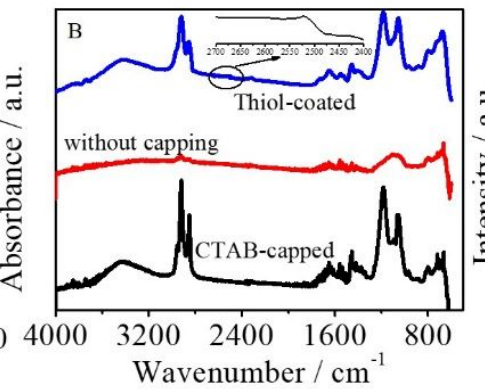

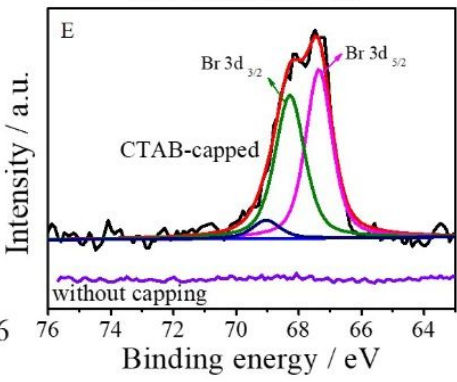

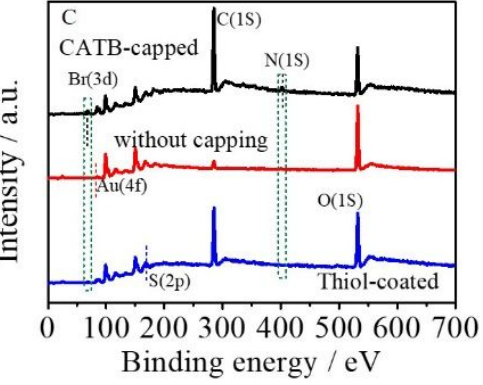

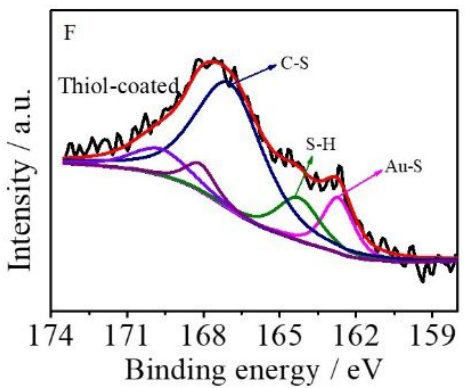

Fig. S6. Spectroscopic and XPS characterization of AuNRs modified by three different media. (A) UV-visible absorbance spectra and (B) ATR-FTIR spectra of CTABstabilized (black line), without stabilizer (red line) and thiol-modified gold nanorods (blue line). (C) Survey scan XPS spectra. (D-E) High resolution XPS of N1s and Br3d (CTAB-stabilized, without stabilizer). (F) S 2p with thiol-modified AuNRs. 

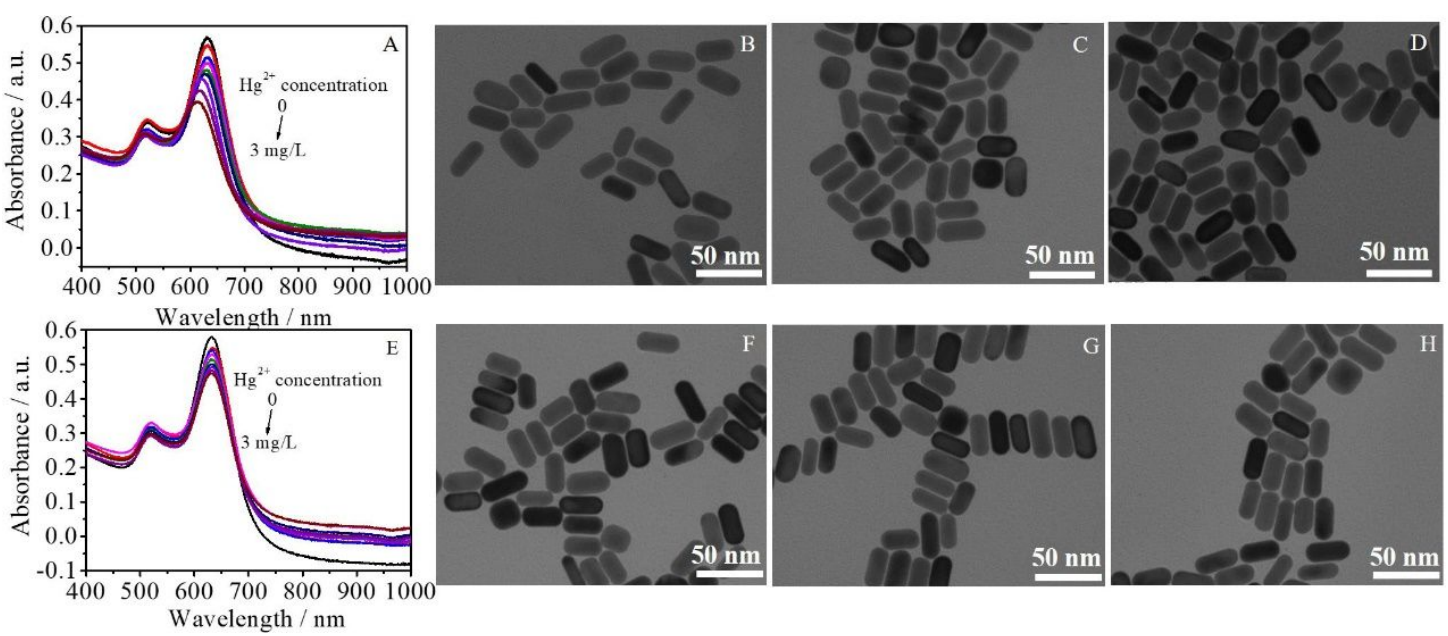

Fig. S7. (A) The extinction spectrum of the AuNRs from the addition of the thiolmodified AuNRs to the $\mathrm{NaBH}_{4}(5 \mathrm{mmol} / \mathrm{L})$ solution containing different volumes of $\mathrm{Hg}^{2+}$. (B-D) TEM images of representative thiol-coated AuNRs obtained under 0, 1.5 $\mathrm{mg} / \mathrm{L}$, and $3 \mathrm{mg} / \mathrm{L} \mathrm{Hg}^{2+}$ treatments. (E) The spectra and morphology changes of the thiol-coated AuNRs under a solution containing $5 \mathrm{mmol} / \mathrm{L}$ AA solution and different volumes of $\mathrm{Hg}^{2+}$. (F-H) TEM images of thiol-coated AuNRs corresponding to different concentrations of $\mathrm{Hg}^{2+}(0,1.5 \mathrm{mg} / \mathrm{L}$, and $3 \mathrm{mg} / \mathrm{L})$ when AA is used as a reducing agent.

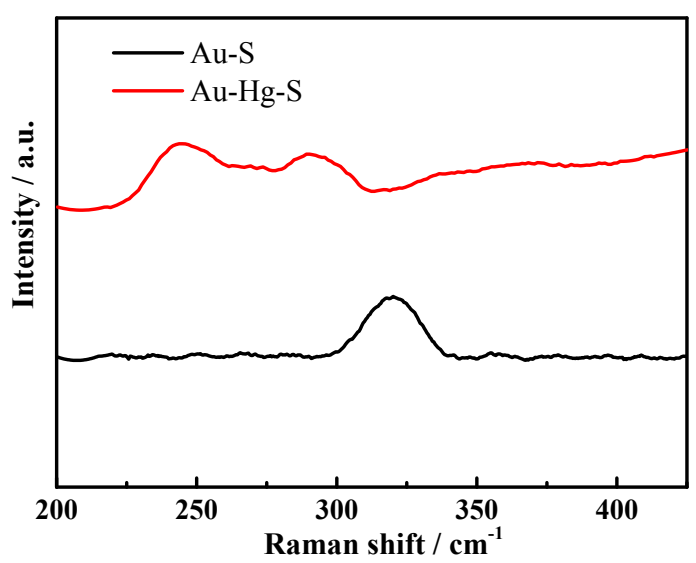

Fig S8. Raman spectra of thiol-modified AuNRs before and after adding Hg. 

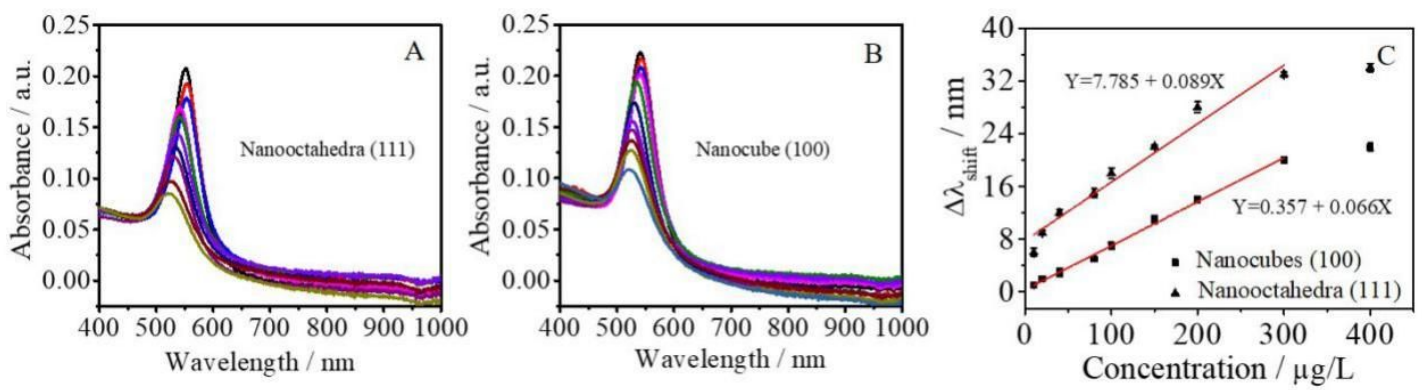

Fig. S9. UV-visible absorption spectra of gold nanooctahedra (A) and gold nanocube (B) with different volumes $(0-2.0 \mathrm{mg} / \mathrm{L})$ of $\mathrm{Hg}^{2+}$ reduced by excess $\mathrm{NaBH}_{4}$. (C) The plot shows the UV-visible absorption shift of gold nanoparticles with different crystal faces as a function of $\mathrm{Hg}^{2+}$ concentration.

Table S1. Measured average zeta-potential during the reaction process of gold nanorods modified with different stabilizers at $25^{\circ} \mathrm{C}$.

\begin{tabular}{ccccc}
\hline \multirow{2}{*}{ AuNRs } & Initial & Intermediate & Final \\
& & state & state & state \\
\hline CTAB-AuNRs & +29.3 & +19.4 & +15.2 \\
Zeta potential & AuNRs-without & -10.2 & -6.4 & -2.1 \\
$(\mathrm{mV})$ & CTAB & & & \\
& SH-AuNRs & +18.1 & +11.4 & +8.6 \\
\hline
\end{tabular}

